\title{
GaN heterostructures with diamond and graphene
}

\author{
B. Pecz, L. Toth, G. Tsiakatouras, A. Adikimenakis, A. Kovacs, M. Duchamp,
} R. E. Dunin-Borkowski, Rositsa Yakimova, P. L. Neumann, H. Behmenburg, B. Foltynski, C. Giesen, M. Heuken and A. Georgakilas

\section{Linköping University Post Print}

N.B.: When citing this work, cite the original article.

Original Publication:

B. Pecz, L. Toth, G. Tsiakatouras, A. Adikimenakis, A. Kovacs, M. Duchamp, R. E. DuninBorkowski, Rositsa Yakimova, P. L. Neumann, H. Behmenburg, B. Foltynski, C. Giesen, M. Heuken and A. Georgakilas, GaN heterostructures with diamond and graphene, 2015, Semiconductor Science and Technology, (30), 11, 114001.

http://dx.doi.org/10.1088/0268-1242/30/11/114001

Copyright: IOP Publishing: Hybrid Open Access

http://www.iop.org/

Postprint available at: Linköping University Electronic Press

http://urn.kb.se/resolve?urn=urn:nbn:se:liu:diva-123529 


\title{
GaN heterostructures with diamond and graphene
}

B. Pécz ${ }^{1}$, L. Tóth ${ }^{1}$, G. Tsiakatouras ${ }^{2}$, A. Adikimenakis ${ }^{2}$, A. Kovács ${ }^{3}$, M. Duchamp ${ }^{3}$, R. E. DuninBorkowski $^{3}$, R. Yakimova ${ }^{4}$, P. L. Neumann ${ }^{1}$, H. Behmenburg ${ }^{5}$, B. Foltynski ${ }^{5}$, C. Giesen ${ }^{5}$, M. Heuken ${ }^{5}$ and A. Georgakilas ${ }^{2}$

${ }^{1}$ Institute for Technical Physics and Materials Science, Centre for Energy Research, Hungarian Academy of Sciences, MTA EK MFA, 1121 Budapest, Konkoly-Thege M. u. 29-33, Hungary

${ }^{2}$ IESL/FORTH and Physics Department/University Crete, P. O. Box 1385, 71110 Heraklion, Greece

${ }^{3}$ Ernst Ruska-Centre for Microscopy and Spectroscopy with Electrons, Peter Grünberg Institute, Forschungszentrum Jülich, D-52425, Germany

${ }^{4}$ Department of Physics, Chemistry and Biology, Linköping University, S-581 83 Linköping, Sweden

${ }^{5}$ AIXTRON SE, Kaiserstrasse 98, 52134 Herzogenrath, Germany

E-Mail: pecz@mfa.kfki.hu

\begin{abstract}
The full performance of GaN devices for high-power applications is not exploited due to the selfheating. Possible solutions are the integration of materials with high heat conductivity i.e., single crystalline diamond and graphene layers. We report the growth of single crystalline (0001)-oriented GaN thin film on (100), (110) and (111) diamond single crystals studied by transmission electron microscopy (TEM) in cross-sections. As for graphene, we show a high-quality GaN layer that was deposited on patterned graphene layers and $6 \mathrm{H}-\mathrm{SiC}$. The atomic structures of the interfaces in the heterostructure are studied using aberration-corrected scanning TEM combined with energy dispersive X-ray and electron energy-loss spectroscopy.
\end{abstract}

\section{Introduction}

Nitride-based (e.g. GaN, InGaN and $\mathrm{AlGaN}$ ) thin films (III-nitrides) are applied not only in various optoelectronic devices and sensors, but also in high power devices, typically in transistors. Concerning their performance, the most difficult technical problem is the extraction of the dissipated heat during their operation. For the III-nitride growth it would be advantageous to use substrates with excellent heat conductivity in order to avoid the self-heating of high power devices. Probably the best 
candidates are diamond and graphene with their superior thermal conductivity $(2000 \mathrm{~W} / \mathrm{mK}$ for diamond and $5000 \mathrm{~W} / \mathrm{mK}$ for graphene). A review paper on the thermal properties of carbon based materials including diamond, graphene and few layer graphene (FLG) can be found in [1]. However, very few III-nitride epitaxial growth experiments are reported on diamond and even less on graphene. Probably the first growth experiment using metal organic chemical vapour deposition (MOCVD) was carried out by Hageman et al. [2] on single crystalline diamond and resulted in poor quality GaN. Dreumel et al. grew also polycrystalline $\mathrm{GaN}$ on nanocrystalline diamond/Si template [3] and later oriented GaN crystals on polycrystalline diamond substrates [4]. The epitaxial growth of $\mathrm{GaN}$ is difficult due to the high lattice misfit in the GaN/diamond system. Recently we have shown that single crystalline (0001) GaN films can be grown on (111) diamond substrates [5]. The thermal management of heterostructures containing graphene or related materials includes lithographically patterned graphene oxide in light-emitting diodes (LEDs) [6]; transferable GaN layers deposited on $\mathrm{ZnO}$ coated graphene layers [7]; thermal heat-escaping channels of graphene layers prepared on the top of $\mathrm{AlGaN} / \mathrm{GaN}$ transistors [8]. We also reported [9], that nitride layers can be grown by MOCVD on patterned graphene/SiC templates.

In the present study, we show the successful growth of high-quality $\mathrm{N}$-face $\mathrm{GaN}$ thin films deposited on (i) single crystalline diamond and (ii) patterned graphene and (0001) 6H-SiC. Both material systems were studied in cross-sectional geometry using conventional transmission electron microscopy (TEM) and aberration-corrected TEM operated at 200 and $300 \mathrm{kV}$. The preparation of electron transparent specimens of diamond and graphene containing samples for TEM characterization represented a significant challenge due to the hardness of diamond and sensitivity of graphene layers to ion energies used during the milling process. We used a conventional mechanical and Ar ion milling (Technoorg-Linda model) process to prepare the diamond samples. Low energy ion milling was carried out on specimens embedded into a Ti supporting ring [10]. The graphene containing crosssections were prepared by focused ion beam (FIB) in a dual-beam system using ion energies of 30 and $5 \mathrm{keV}$. A Pt/C protective layer was deposited onto the surface of the $\mathrm{GaN}$ using the electron and ion beams with thicknesses of 0.5 and $2.5 \mu \mathrm{m}$, respectively. The FIB lamella was fixed onto a standard $\mathrm{Cu}$ Omniprobe grid using Pt/C layer deposition. The ion beam-induced surface damage was then reduced by using low-energy $(0.5 \mathrm{keV})$ focused Ar-ion milling at an incident angle of $10^{\circ}$ in a Fischione Nanomill 1040 system.

Bright-field (BF), dark-field images and selected area electron diffraction (SAED) patterns were taken in a Philips CM 20 microscope working at $200 \mathrm{keV}$. High-resolution TEM studies were done using a JEOL 3010 and FEI Titan electron microscopes at $300 \mathrm{keV}$.

\section{Single crystalline GaN on diamond}


In the first part of this paper we report on the microstructure of $\mathrm{GaN}$ thin films that were grown on (100) and (110) diamond single crystal substrates by plasma-assisted molecular beam epitaxy (PAMBE), employing a nitrogen radio frequency (RF) plasma source [11]. Then we will discuss the nitride layers grown by MOCVD on patterned FLG/SiC. Cross-sectional TEM studies showed that pre-treatments (nitridation) of the diamond surface with the nitrogen beam improved the structural quality of the grown epilayers by reducing the threading dislocation density and eliminated the formation of inversion domains. The chemical mapping on nitride/FLG/SiC structure was carried out by energy dispersive X-ray spectroscopy (EDXS).

Single crystalline diamond substrates with sizes of approximately $5 \times 5 \mathrm{~mm}^{2}$, prepared by Element6 (www.e6.com) were used. GaN thin films with thickness of about $1 \mu \mathrm{m}$ were grown on the single crystal diamond substrates by Plasma-Assisted MBE (PAMBE). Two sets of experiments were carried out: In the first set, a thin AIN nucleation layer with thickness of approximately $15 \mathrm{~nm}$ was directly grown on diamond, at $780{ }^{\circ} \mathrm{C}$, under stoichiometric growth conditions, without any prior treatment of the diamond surface, followed by the thick GaN layer, grown at $700{ }^{\circ} \mathrm{C}$, grown under metal-rich conditions. In the second set of experiments, the diamond surface was exposed to the nitrogen beam (nitridation), and then an AlN nucleation layer, with thickness of about $100 \mathrm{~nm}$ was grown, under the same growth conditions as the first set, followed by the thick GaN layer. The temperature of the nitridation was varied from $125^{\circ} \mathrm{C}$ to $750{ }^{\circ} \mathrm{C}$, while the duration time was varied from 1 minute to 60 minutes.

The optimal conditions for the nitridation treatment of the diamond substrates were selected according to our previous experience for the effects of exposure to the nitrogen plasma source beam of sapphire [12] and GaAs substrates [13, 14]. The nitrogen plasma beam can be very reactive and may result to roughening of the substrate surfaces at high substrate temperature. In the case of sapphire substrates, it was found that low temperature nitridation did not result to formation of interfacial AlN layer but improved the smoothness of the substrate surface and the abruptness of the substrate/epilayer interface. This is attributed to removal of contaminants from the substrate surface by reaction with the active species of the nitrogen plasma source. However, a sufficiently long time, such as 60 min may be needed to secure the cleaning of the substrate surface at low temperature.

Figure 1 (a) shows the cross section of the GaN film deposited on (110) diamond, without the nitridation treatment. (The surface roughness of the GaN layer should not be considered here, since it is a result of the chemical etching carried out in order to determine the polarity of the grown layers). A large number of threading dislocations, as well as inversion domains (ID), were formed in the GaN layer. The selected area electron diffraction pattern (SAED) in Fig. 1 (b) revealed the single crystalline growth of the III-nitride layers on diamond $\{110\}$ substrate, with an epitaxial relationship of (0002)GaN//(022)diamond and (1010)GaN//(400)diamond. 

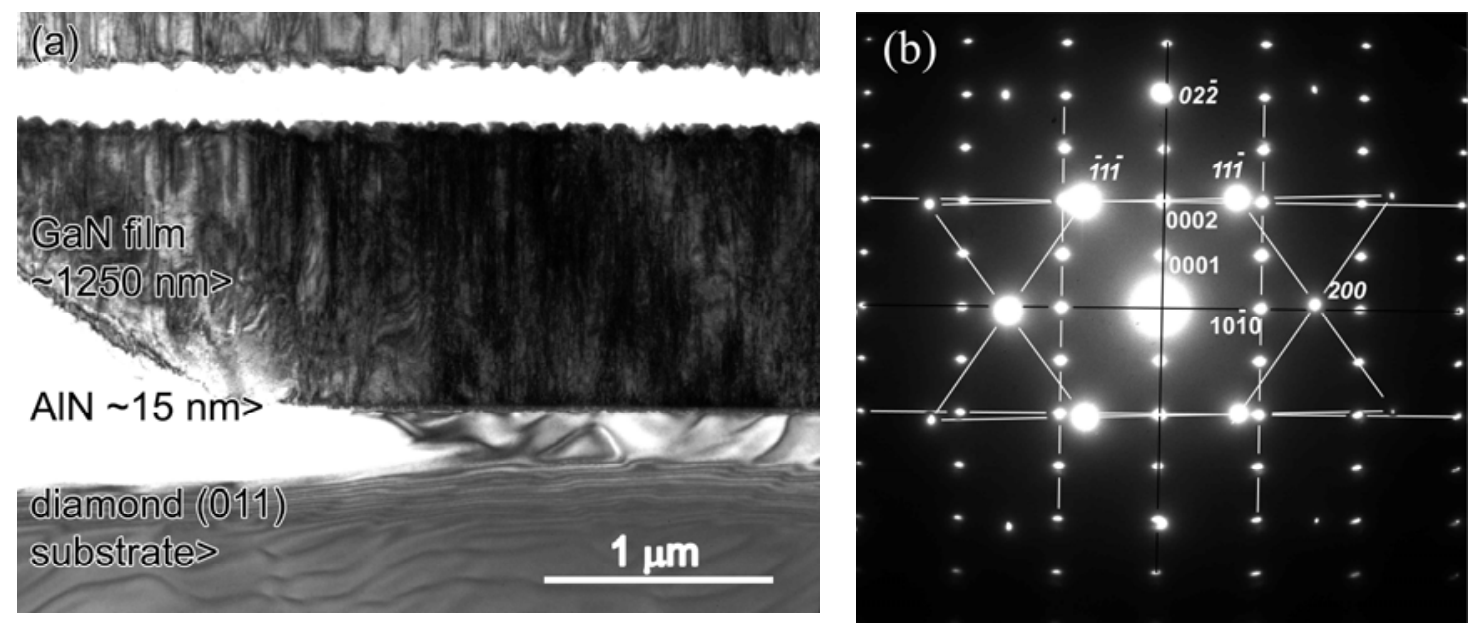

Figure 1. Microstructure of GaN/AlN (0001) layers on diamond (110) substrate without nitridation treatment. (a) BF TEM image of the GaN film showing the high density of IDs in GaN layer (b) SAED pattern of the nitride layers and diamond. The reflections of hexagonal nitride layers and cubic diamond are marked by four and three digit indices, respectively. 


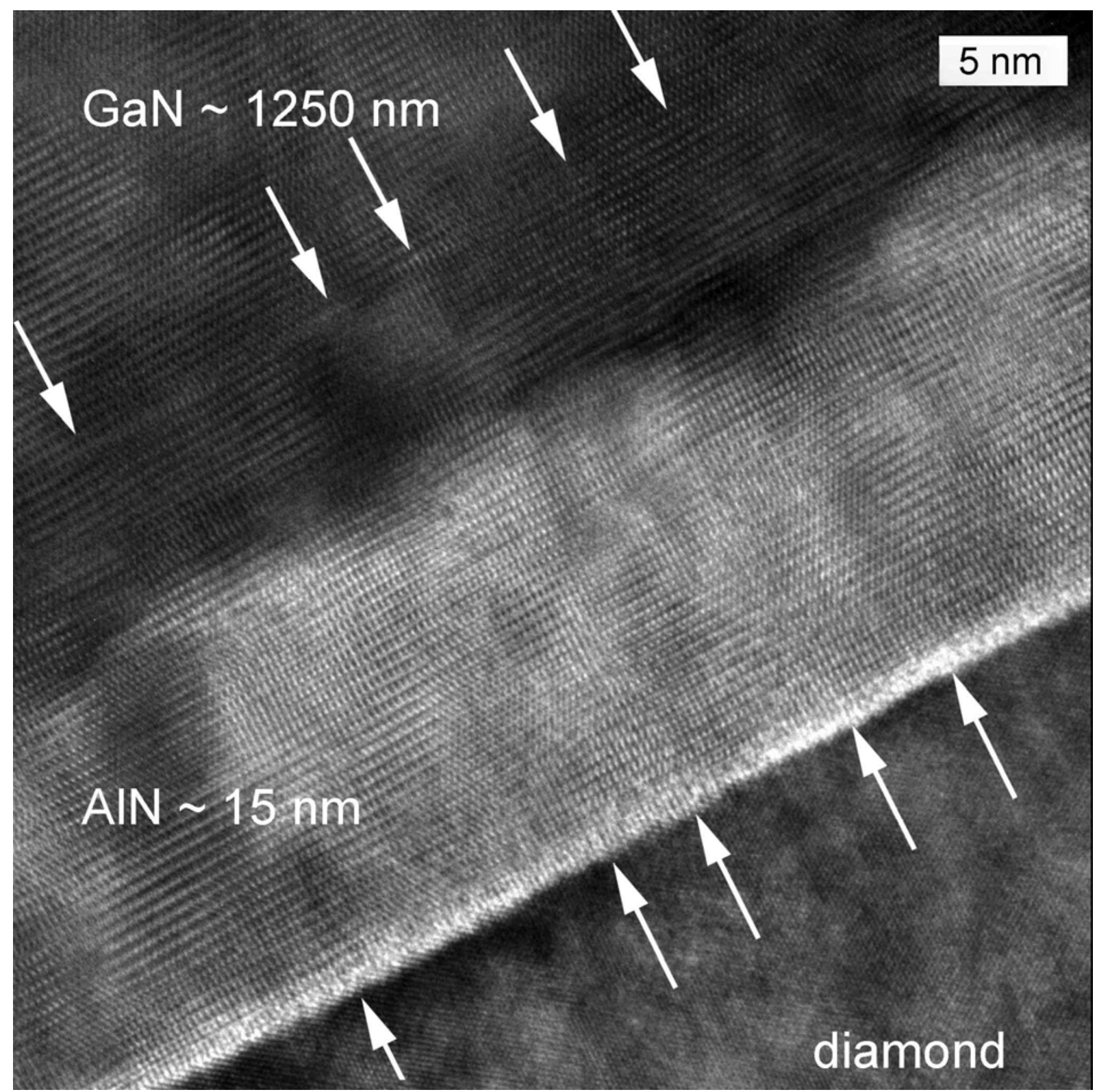

Figure 2. High-resolution TEM image of the GaN/AIN/diamond interfacial region showing a highdensity of IDs (some of them are marked by arrows) in the GaN/AIN layers. The IDs formed already on the surface of diamond.

The high-resolution TEM study of the GaN/AlN/diamond interfacial region (shown in Figure 2) revealed a high-density of IDs that formed already at the AlN/diamond interface. Those IDs, then, propagated to the top surface of the nitride layers. Although the grown nitride layers are epitaxial to the diamond substrate the large number of defects, especially the high density of IDs, would limit the device applications.

Figure 3 shows the overview of the nitride structure, that was grown on (110) diamond substrate, nitridated prior to the AlN growth for 60 minutes at $150{ }^{\circ} \mathrm{C}$. The AlN nucleation layer was $90 \mathrm{~nm}$ thick. It was found that the thick GaN layer is free of IDs, and only threading dislocations could be 
observed. Thus, we can conclude that the nitridation of the diamond surface, prior to the growth initiation, significantly affects the formation of crystal defects such as threading dislocations and IDs. Single polarity GaN films were achieved on the nitridated diamond substrates. The polarity of the layer was determined by convergent beam electron diffraction which proved the nitrogen polarity of the layers. Finaly, in order to obtain a 2DEG heterostructure, a thin AlN layer was grown on top of the thick GaN layer, followed by a $30 \mathrm{~nm} \mathrm{GaN} \mathrm{cap} \mathrm{layer.} \mathrm{Those} \mathrm{layers} \mathrm{are} \mathrm{uniform} \mathrm{and} \mathrm{flat} \mathrm{(Fig.} \mathrm{3b).}$
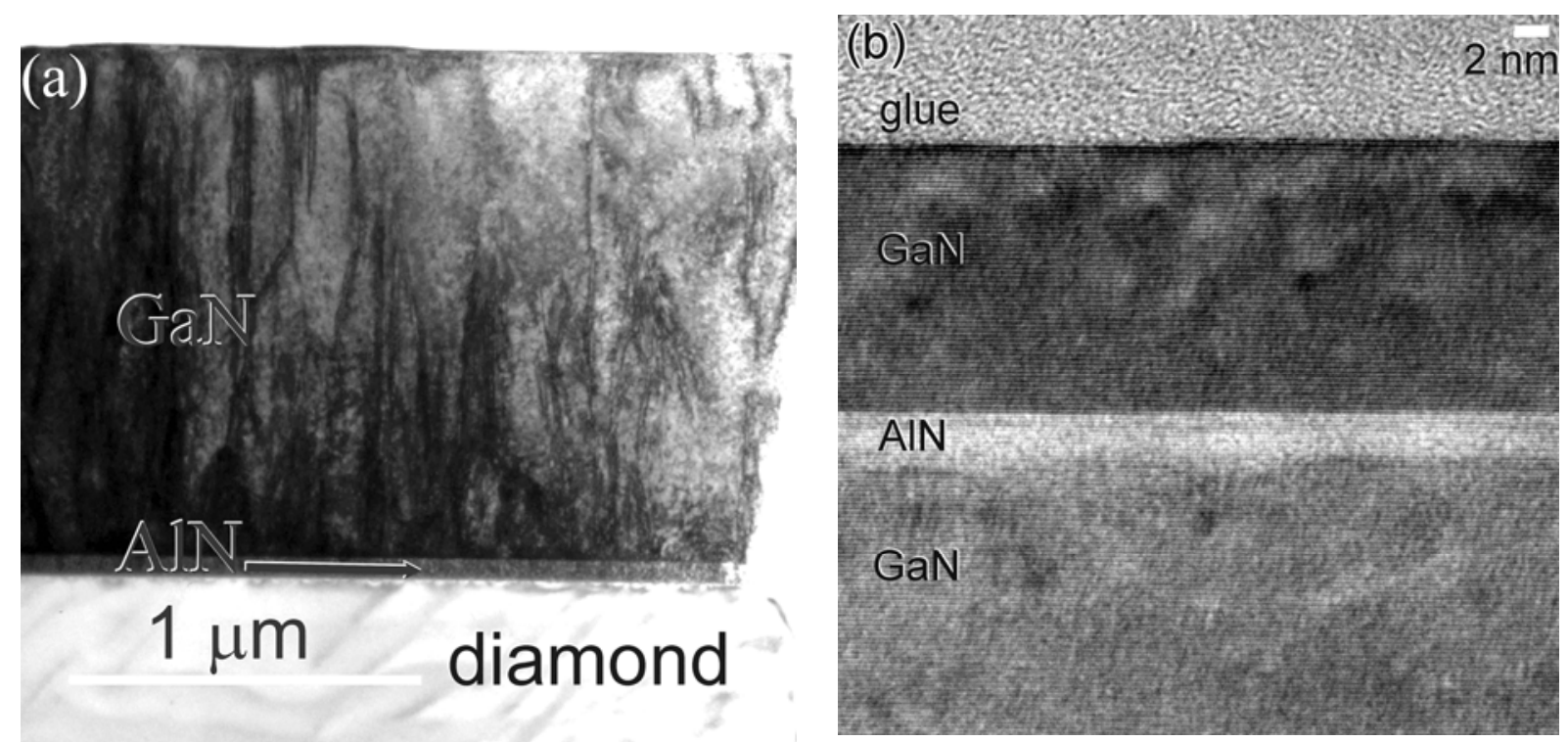

Figure 3. GaN / AlN layers grown on nitrided diamond (110) surface. (a) BF TEM image. (b) highresolution TEM image showing active layers in the top region.

In our earlier work on (111) diamond [5] we made a detailed high resolution analysis of the interface between the nitride and diamond and found no additional phase(s) on the diamond surface after nitridation. Most probably the nitridation could clean the surface of diamond by the active nitrogen species, like it was shown for $\mathrm{NH}_{3}$ in the case of $\mathrm{SiC}$ [15]. 


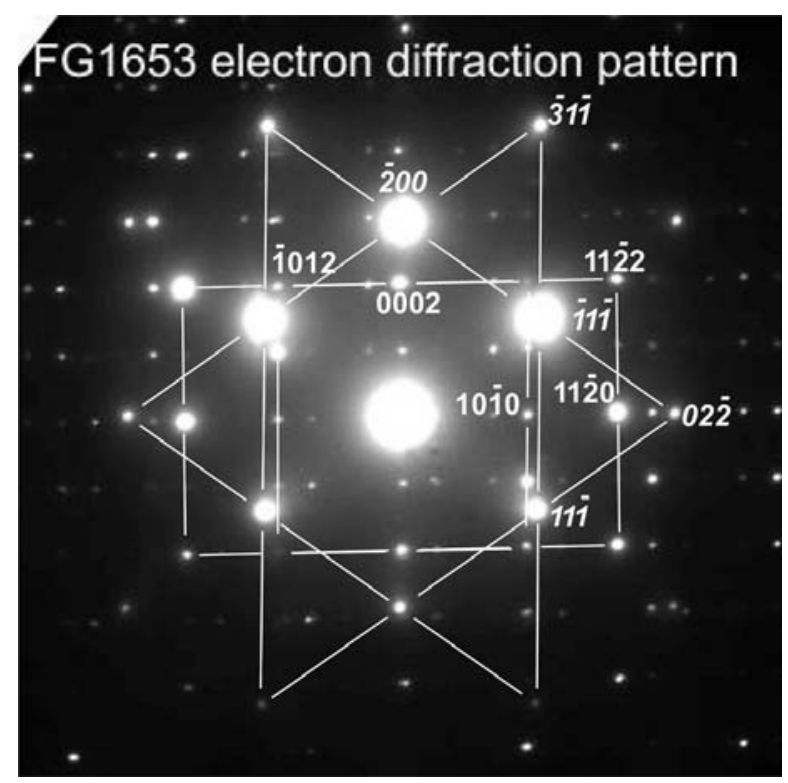

Fig. 4. Selected area diffraction pattern of GaN grown on (001) diamond.

On (001) oriented diamond two different domains of GaN were grown with a common c-axis parallel to the surface normal. Epitaxial relationships are the following: (0002)GaN//(400)diamond and $(11 \underline{2} 0) \mathrm{GaN} / /(02 \underline{2})$ diamond, or $(10 \underline{10}) \mathrm{GaN} / /(02 \underline{2})$ diamond. Knowing that the epitaxial configuration on (111) diamond [5] is the same as in the case of (110) we can conclude, that on all three orientations the grown GaN film is c-axis oriented.

\section{GaN grown on patterned graphene}

The integration of graphene into III-nitride thin films is very challenging due to the lack of chemical reactivity between graphene and the growing nitride (only polycrystalline material could be received with very weak bonding when we carried out an MOCVD growth of nitride layers on SiC completely covered by graphene). Recently, we proposed a simple patterning process [9] of graphene layers grown on $6 \mathrm{H}-\mathrm{SiC}$ substrate using polymethyl methacrylate (PMMA) and electron beam lithography prior to the standard nitride growth using MOCVD, as shown in Fig. 5 (a and b). The unmasked graphene layers were removed by high-density plasma etching using an $\operatorname{Ar}(89 \%)$ and $\mathrm{O}_{2}(11 \%)$ mixture. The PMMA mask was dissolved by acetone. The patterned structure allowed to nucleate AlN directly on bare $\mathrm{SiC}$ with strong bonding and transfering the epitaxial orientation to the regions growing above the graphene. The graphene layers were prepared on the Si-terminated side of 0001 oriented $6 \mathrm{H}-\mathrm{SiC}$ single crystals by high temperature sublimation technique [16]. Figure 5 (c) shows a BF TEM image of the heterostructure. The dislocation density in the GaN layer is $\sim 10^{9} \mathrm{~cm}^{-2}$. The GaN layer forms a smooth surface and contains IDs that reach the surface forming a little pit. The detailed analysis of the interface between the graphene layers and the $6 \mathrm{H}-\mathrm{SiC}$ showed that the chemical etching of the lithographically patterned graphene/SiC removed not only the graphene from the window 
regions, but a few layers of topmost $6 \mathrm{H}-\mathrm{SiC}$. The IDs were formed in $\mathrm{GaN}$ grown directly on $6 \mathrm{H}-\mathrm{SiC}$, as shown in Fig. 5 (c). It is striking that the GaN layer is defective above the intact graphene layers, with a crystallographic orientation that is different from that of the single crystalline GaN above the graphene free $\mathrm{SiC}$ stripes. The single-crystalline GaN overgrows the poly-crystalline GaN by selfassisted lateral epitaxial overgrowth, thus reducing the dislocation density.

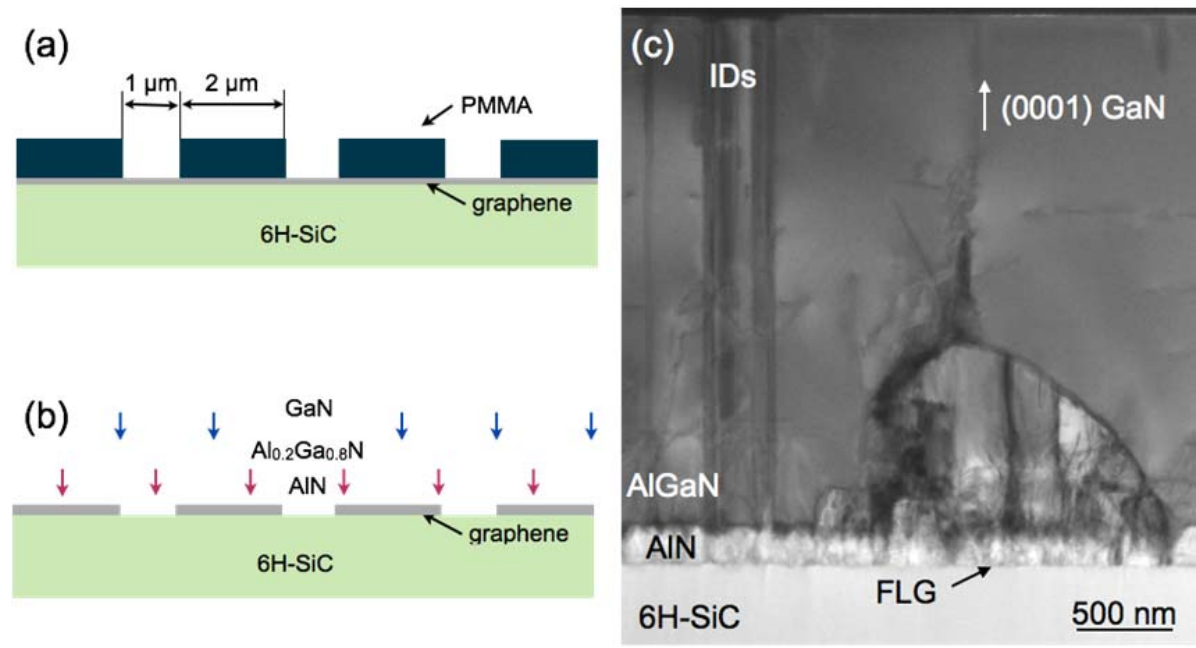

Figure 5. a, b) Schematic figure of the patterning process and growth. c) Low magnification BF TEM overview of the heterostructure. FLG and ID indicate the few layer of graphene and inversion domain, respectively.
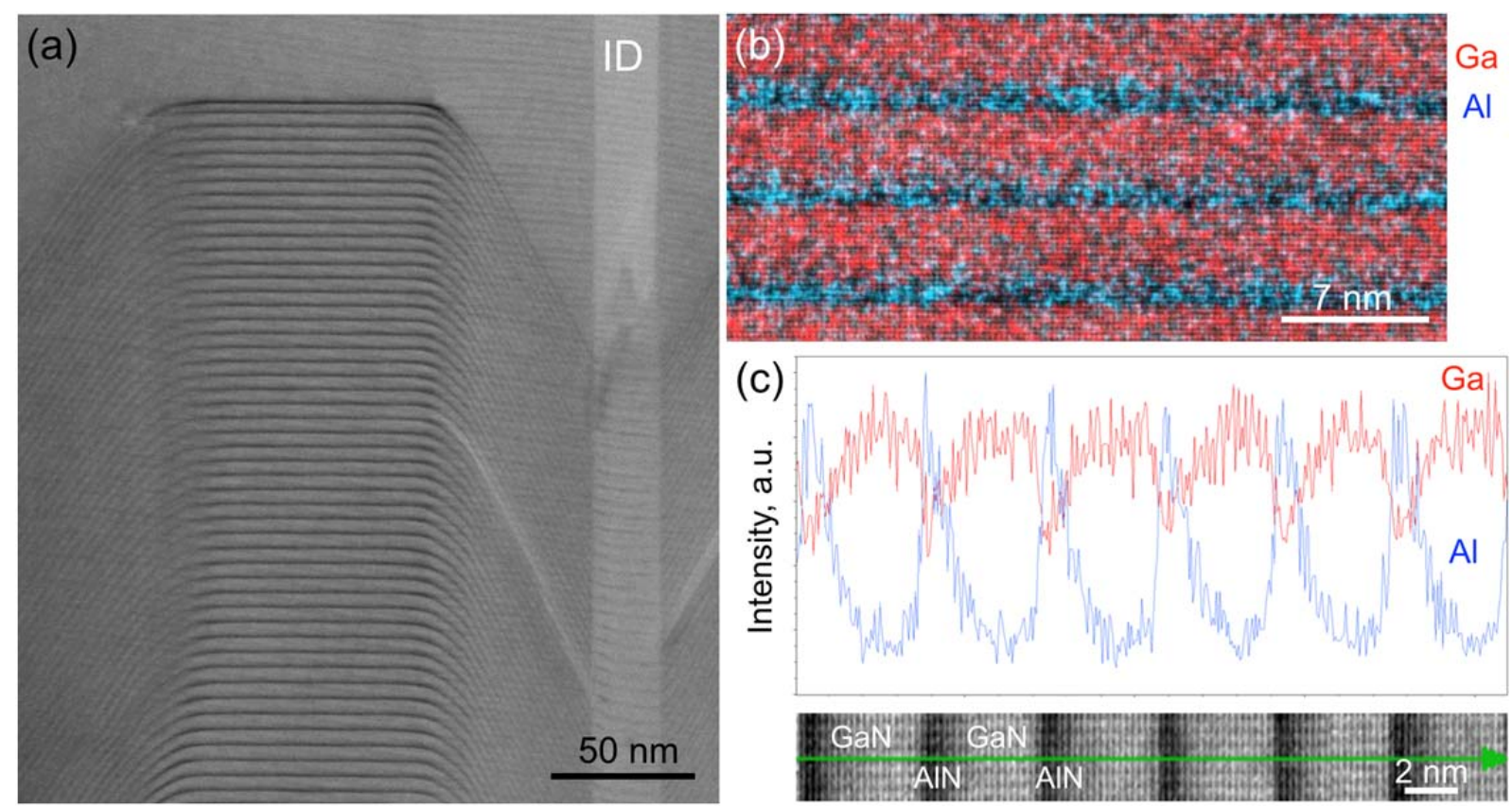

Fig. 6. (a) HAADF STEM image of the AIN/GaN superlattice formed between the AlN buffer layer and GaN. (b) HAADF STEM image overlapped with Ga and Al distribution extracted from EDXS spectrum imaging experiment. The inner ADF detector semi-angle used was $69 \mathrm{mrad}$. (c) The distribution of $\mathrm{Ga}$ and $\mathrm{Al}$ across the layers extracted from EDXS and the corresponding HAADF 
The AlN buffer layer (Fig. 5c) is rough and the morphology suggests that it was nucleated and grown over the graphene in a $3 \mathrm{D}$ way. Therefore it is not surprising, that the $\mathrm{AlGaN}$ layer grown on the buffer is also composed from hillocks (Fig. 6a). However, our electron microscopy study revealed the fact that this layer, which is a homogeneous and flat ternary layer in the control sample (without graphene) is decomposed to superlattices. The analytical investigations (Fig. $6 \mathrm{~b}$ and c) show, that the superlattice layers are pure AlN and GaN layers. Also the thickness of the individual AlN (1 - 1.2 $\mathrm{nm}$ ) and $\mathrm{GaN}$ layers $\left(3.6 \mathrm{~nm}\right.$ ) fits to the available amount of the metals in the nominal $\mathrm{Al}_{0.2} \mathrm{Ga}_{0.8} \mathrm{~N}$ composition. The formation mechanism of the $\mathrm{AlN} / \mathrm{GaN}$ superlattices is not completely understood. However, most probably this is connected to some carbon species originating from the graphene layers and being present in the "ternary" layer. Similar effect was observed, when low amount of carbon was introduced into carbon-indium co-doped GaAs grown by MOCVD [17].

\section{Conclusions:}

In conclusion, GaN thin films of high structural quality were grown on (110) single crystal diamond substrates by PAMBE using a 15-90 nm AlN nucleation layer. The optimized growth samples exhibited a single epitaxial relationship of $\mathrm{GaN}$ (0001) on diamond (110) with single N-face polarity. The formation of inversion domains was successfully eliminated by introducing a diamond nitridation treatment. This solution is effective for the heat dissipation problem of GaN based HEMTs as it was proved by Alomari et al. [18]. The second solution based on graphene layers is also very promising and thermal reflectance measurements are in progress to prove that.

\section{Acknowledgements}

This work is supported by OTKA project (Hungary) No. K 108869. The authors acknowledge financial support from the European Union under the Seventh Framework Program under a contract for an Integrated Infrastructure Initiative. Reference 312483 - ESTEEM2.

\section{References}

1) A.A. Balandin: Nature Materials, 10 (2011) 569.

2) P.R. Hageman, J.J. Schermer, P.K. Larsen: Thin Solid Films, 143 (2003) 9.

3) G.W.G. van Dreumel, J.G. Buijnsters, T. Bohnen, J.J. ter Meulen, P.R. Hageman, W.J.P. van Enckevort, E. Vlieg: Diamond and Relat. Mater. 18 (2009) 1043.

4) G.W.G van Dreumel, T. Bohnen, J.G. Buijnsters, W.J.P. van Enckevort, J.J. ter Meulen, P.R. Hageman, E. Vlieg: Diamond and Relat. Mater. 19 (2010) 437.

5) B. Pécz, L. Tóth, Á. Barna, G. Tsiakatouras, A.O. Ajagunna, A. Kovács, A. Georgakilas: Diamond and Related Materials, 34 (2013) 9-12. 
6) N. Han, T.V. Cuong, M. Han, B.D. Ryu, S. Chandramohan, J.B. Park, J.H. Kang, Y.J. Park, K. B. Ko, H.Y. Kim, H.K. Kim, J.H. Ryu, Y.S. Katharria, C.J. Choi, C.H. Hong: Nat. Comm., 4 (2013) 1452.

7) K. Chung, K.C.-H. Lee and G.C. Yi: Science, 330 (2010) 655.

8) Z. Yan, G. Liu, J.M. Khan, A.A. Balandin: Nat. Comm., 3 (2012) 827.

9) A. Kovács, M. Duchamp, R.E. Dunin-Borkowski, R. Yakimova, P. L. Neumann, H. Behmenburg, B. Foltynski, C. Giesen, M. Heuken and B. Pécz: Advanced Materials Interfaces, 2 (2015) 1400230.

10) Á. Barna, G. Radnóczi and B. Pécz, in Handbook of Microscopy, (eds. S. Amelinckx, D. van Dyck, J. van Landuyt, G. van Tendelo) VCH Verlag, Vol. 3, Chapter II/3, (1997) 751.

11) E Iliopoulos, A Adikimenakis, E Dimakis, K Tsagaraki, G Konstantinidis, A Georgakilas: J. Cryst. Growth 278 (2005) 426.

12) S. Mikroulis, A. Georgakilas, A. Kostopoulos, V. Cimalla, E. Dimakis and Ph. Komninou, Appl. Phys. Lett. 80 (2002) 2886.

13) A. Georgakilas, Zs. Czigany, K. Amimer, V. Yu. Davydov, L. Toth and B. Pecz, Mater. Sci. Eng. B 82 (2001) 16.

14) A. Georgakilas, K. Amimer, P. Tzanetakis, Z. Hatzopoulos, M. Cengher, B. Pecz, Zs Czigany, L. Toth, M.V. Baidakova, A.V. Sakharov and V.Yu. Davydov, J. Crystal Growth 227-228 (2001) 410 .

15) A.J McGinnis, D. Thomson, R.F. Davis, E. Chen, A. Michel, H.H. Lamb: J. Cryst. Growth 222, (2001) 452 .

16) R. Yakimova, C. Virojanadara, D. Gogova, M. Syväjärvi, D. Siche, K. Larsson, L. I. Johansson: Mater. Sci. Forum Vols. 645-648 (2010) 565.

17) M.A. di Forte-Poisson, A. Roman, S. Bernard, J. Humbert, L.Teisseire, B. Pecz, A.Sulyok, J. di Persio, 7th European Workshop on Metal-Organic Vapour Phase Epitaxy and Related Growth Techniques, Berlin, Germany June 8 -11, 1997

18) M. Alomari, A. Dussaigne, D. Martin, N. Grandjean, C. Gaquiere, E. Kohn: Electronic Lett., 46 (2010) 299. 